\title{
A cross-sectional examination of medicinal substance abuse and use of nonmedicinal substances among Canadian youth: findings from the 2012-2013 Youth Smoking Survey
}

\author{
Cesar Leos-Toro BSc, David Hammond PhD, Stephen Manske EdD
}

Abstract

Background: Medicinal substance abuse is prevalent in Canada; however, little is known about patterns of abuse among young people. In this study, we sought to characterize the abuse of medicinal substances, such as prescription medications and selected over-thecounter substances, as well as that of licit and illicit nonmedicinal substances, using a nationally representative sample of young people.

Methods: Cross-sectional, nationally representative data for children in grades 7-12 were obtained from Health Canada's 20122013 Youth Smoking Survey $(n=38$ 667). Multinomial regression analyses were conducted to examine subgroup differences in medicinal substance abuse and comorbid abuse of both medicinal and nonmedicinal substances.

Results: About $5 \%$ of youth reported abusing medicinal substances in the previous year. Dextromethorphan, a substance found in many cough and cold syrups, was the most widely abused (2.9\%), followed by pain medications (2.6\%), sleeping medications (1.8\%), stimulants (1.7\%) and sedatives (1.0\%). Abuse of nonmedicinal substances aside from tobacco and alcohol was reported by $21.3 \%$ of the population, and abuse of any substances was detected in $23.0 \%$ of the surveyed population. Girls at each grade level reported higher rates of abuse of medicinal substances than boys. Regional differences were seen with regard to the types of substances abused across Canada.

Interpretation: A substantial minority of Canadian youth report abusing medicinal substances, including over-the-counter medications (e.g., cough syrup) and prescriptions medications (e.g., pain medication). In contrast to nonmedicinal substances, girls were more likely than boys to report abuse of medicinal substances.

ontrolled substance abuse has been associated with numerous economic, social and health consequences. ${ }^{1-6}$ Controlled substances are drugs that have been identified by the Canadian federal government as having an elevated potential for abuse or addiction, and are listed in schedules I to V of Canada's 1996 Controlled Drugs and Substances Act. ${ }^{7}$ Abuse of controlled medication refers to the consumption of substances intended for medical treatment for the purpose of producing euphoric feelings of well-being (a "high"), whereas misuse refers to a deviation from prescribed instructions, such as taking an additional sleeping pill when the first did not have the desired effect. ${ }^{8}$ Comprehensive cost analyses of controlled substance abuse have yet to be conducted in Canada since one was undertaken in 2006 with limited data from 2002; however, medicinal substance abuse is known to contribute considerably to the annual estimated costs related to substance abuse calculated at $\$ 40$ billion. ${ }^{9,10}$ Medicinal substances include substances listed in the Controlled Drugs and Substances Act as well as substances used for therapeutic purposes such as cough syrups or sleeping medications.

Prescription opioids are the most commonly abused type of medicinal substances. Prescription opioid abuse ranks third in terms of substance use burden after tobacco and alcohol; these measures are based on the best available evidence and rely greatly on variable provincial and locally captured indicators as they relate to mortality, morbidity and diversion of substances. ${ }^{11}$ About $80 \%$ of prescription opioids are dispensed in community settings without postdispensing control mechanisms, allowing for increased supply of these substances in homes across the country. ${ }^{12}$ Young Canadians are particularly vulnerable to prescription opioid abuse and other substances available over the counter; however, there are gaps in knowledge in characterizing the abuse of these substances. ${ }^{13}$ According to available data from the 2010-2011 Ontario Student Drug Use and Health Survey, prescription opioid abuse is the most commonly reported controlled medication abused among Ontario youth, with rates of nonmedicinal use in the past year ranging from $15 \%$ to $20 \%$ of secondary students, compared with $6 \%$ of the province's general adult population. ${ }^{14,15}$

Competing interests: None declared.

This article has been peer reviewed.

Correspondence to: David Hammond, dhammond@uwaterloo.ca CMAJ Open 2015. DOI:10.9778/cmajo.20140094 
Other medications liable to abuse include benzodiazepines and stimulants. ${ }^{16}$ Dextromethorphan, a synthetic opioid in many antitussives (i.e., cold medications), is not currently listed in Canada's Controlled Drugs and Substances Act but has been found to lead to mania or psychosis at elevated levels of consumption. ${ }^{17-20}$ Recent estimates in Ontario suggest an increase in recreational use of dextromethorphan, from $7 \%$ to $10 \%$ among students in grades 7 through 12 from 2011 to 2013. ${ }^{21}$ These rates are consistent with higher levels of stimulant use among young Canadians than in the general adult population. ${ }^{6}$

Youth is a critical period for substance use. Adolescence marks a crucial time of growth and change in neural regions that influence impulsivity and potentially destructive behaviours such as substance abuse. ${ }^{22-25}$ Abuse of controlled substances has been associated with short- and long-term psychological and physiologic health effects, including death, ${ }^{26-29}$ and patterns of substance abuse and misuse among youth predict use in adulthood. ${ }^{30-32}$ To date, however, there are no national estimates of medicinal substance abuse among Canadian young people.

We sought to characterize rates of abuse of medicinal substances and use of nonmedicinal substances using a nationally representative sample of youth. This study examined prevalence of abuse among Canadian youth by sociodemographic factors, concurrent substance use behaviours and region of residence.

\section{Methods}

\section{Participants}

Cross-sectional data were obtained from the 2012-2013 Youth Smoking Survey, which involved 38667 participants from grade 7 to grade 12 in 450 schools from all Canadian jurisdictions excluding Manitoba, Yukon, Northwest Territories and Nunavut. Manitoba's decision to decline participation was based on factors related to separate provincial initiatives not specific to the survey's content or methodology. Comparative analyses from 2010-2011 survey indicate no significant differences in national estimates with and without Manitoba. The survey included Canadian residents attending private, public and Catholic schools, with the exception of youth who were residents of institutions at the time of the survey, students who were living on First Nations Reserves or students who were attending special schools (e.g., schools for the hearing or visually impaired), virtual schools, daycares or schools on military bases.

\section{Design}

The 2012-2013 Youth Smoking Survey was based on a stratified single-stage design. Stratification was based on health region smoking rate and whether the school was an elementary or secondary school. Lists of schools were divided into 2 strata based on smoking rates of students aged 15-19 years within the health region determined by current Canadian Community Health Survey data and the school's postal code in all provinces except Quebec, Ontario and Alberta, where schools were divided into 3 strata. The third stratum acknowledged the size of major metropolitan areas (Montréal,
Calgary/Edmonton and Toronto) and ensured representative samples from these centres. Detailed information on the sample design, methods and survey rates for this wave of survey data are available through Health Canada, as well as the survey's website (www.yss.uwaterloo.ca). ${ }^{33,34}$ The University of Waterloo Human Research Ethics Committee and Health Canada's Research Ethics Board reviewed all necessary protocols and materials before the survey's implementation.

\section{Measures}

The 2012-2013 Youth Smoking Survey collected information on sex, school grade, ethnicity, spending money, region of residence, tobacco use and alcohol bingeing, as well as information about different substances deemed commonly abused by young people (see Appendix 1, available at www.cmajopen. $\mathrm{ca} /$ content/3/4/E387/suppl/DC1).

Ethnicity was assessed by asking "How would you describe yourself? (Mark all that apply)." Responses were categorized as White, Aboriginal (First Nations, Métis, Inuit), Asian, Black, Latin or Other.

"Never smokers" reported that they had not "ever tried cigarette smoking, even just a few puffs"; "Ever smokers" were those who had tried a cigarette as indicated by answering the questions "Have you ever tried cigarette smoking, even just a few puffs?" and, "Have you ever smoked 100 or more whole cigarettes in your life?"; "Experimental smokers" reported that they had tried smoking, even just a few puffs, but reported smoking fewer than 100 whole cigarettes in their lives.

Binge drinking was determined by asking respondents "In the last 12 months, how often did you have a drink of alcohol that was more than just a sip?" and "In the last 12 months, how often did you have 5 drinks of alcohol or more on one occasion?"

Marijuana use was assessed based on the way respondents answered "In the last 12 months, how often did you use marijuana or cannabis?" and was validated using an additional question, "How old were you when you first used marijuana or cannabis?"

Information on medicinal substance and nonmedicinal substance abuse was drawn from the last section of the survey: the response to the question "If you have ever used or tried any of the following drugs, mark the age at which you first used or tried. Then mark if you have used or tried the drug in the last 12 months," and a subsequent probe that asked participants if they had used the listed substances "to get high and not for medical purposes."

\section{Statistical analysis}

All analyses were performed with SAS Software for Windows version 9.4 (SAS Institute Inc., Cary, NC), and all results represent weighted data. The development of the survey weights was accomplished in 2 stages. In the first stage, a weight $\left(W_{1 j}\right)$ was created to account for school selection within health region and school strata. A second weight $\left(W_{2 \mathrm{jg}}\right)$ was calculated to adjust for student nonresponse. The weights were calibrated to the province, sex and grade distribution so that the total of the survey weights by sex, grade and province would equal the actual enrolments in those groups. Finally, bootstrap weights 
were applied to estimate and account for sampling error, including clustered data within schools. The bootstrap weights were constructed separately for each province; a comprehensive description of their construction may be found in accompanying survey literature. ${ }^{33}$ Multinomial logistic regression models were fitted to examine correlates of medicinal, nonmedicinal and concurrent use of both medicinal and nonmedicinal substance abuse (where $0=$ no substance abuse, $1=$ medicinal substance abuse, $2=$ nonmedicinal substance abuse and $3=$ concurrent abuse of medicinal and nonmedicinal substances). Six variables were included in the model that had been previously associated with substance use among Canadian youth: sex, school grade, ethnicity, region of residence, spending money, smoking status and a positive response to alcohol bingeing. ${ }^{35}$ Accepted statistical significance of results was set at $p<0.05$, and a Bonferroni correction was applied to reduce risk of type 1 error.

\section{Results}

\section{Response rate}

The response rate across Canada was $71.7 \%$ and ranged from $58.9 \%$ in Ontario to $91.5 \%$ in Quebec. At the student level, nonresponse was primarily due to parental

Table 1: Characteristics of students (grades 7-12) who participated in the 2011-2012 Youth Smoking Survey $(n=38$ 667)

\begin{tabular}{|c|c|c|c|c|c|c|c|c|c|c|c|c|c|c|c|}
\hline \multirow[b]{3}{*}{ Characteristic } & \multicolumn{15}{|c|}{ Students, \% } \\
\hline & \multirow[b]{2}{*}{ Total } & \multicolumn{2}{|c|}{ All grades } & \multicolumn{2}{|c|}{ Grade 7} & \multicolumn{2}{|c|}{ Grade 8} & \multicolumn{2}{|c|}{ Grade 9} & \multicolumn{2}{|c|}{ Grade 10} & \multicolumn{2}{|c|}{ Grade 11} & \multicolumn{2}{|c|}{ Grade 12} \\
\hline & & Female & Male & Female & Male & Female & Male & Female & Male & Female & Male & Female & Male & Female & Male \\
\hline \multicolumn{16}{|l|}{ Ethnicity } \\
\hline White & 65.1 & 65.2 & 65.1 & 65.2 & 66.2 & 63.1 & 64.4 & 67.7 & 66.0 & 65.9 & 66.2 & 67.2 & 65.4 & 61.9 & 61.9 \\
\hline Asian & 10.4 & 11.0 & 9.8 & 12.2 & 13.0 & 12.8 & 11.4 & 8.7 & 7.9 & 10.3 & 9.2 & 8.2 & 7.8 & 13.9 & 9.8 \\
\hline Black & 5.8 & 5.3 & 6.3 & 5.2 & 3.8 & 6.0 & 5.1 & 4.6 & 7.9 & 5.7 & 6.3 & 4.3 & 7.4 & 5.8 & 7.1 \\
\hline $\begin{array}{l}\text { Aboriginal (First } \\
\text { Nations, Métis, } \\
\text { Inuit) }\end{array}$ & 4.2 & 4.1 & 4.3 & 3.2 & 3.7 & 4.3 & 3.8 & 4.3 & 4.7 & 4.3 & 4.5 & 4.3 & 4.6 & 4.3 & 4.4 \\
\hline $\begin{array}{l}\text { Latin American/ } \\
\text { Hispanic }\end{array}$ & 2.0 & 2.0 & 2.1 & 1.5 & 2.2 & 1.9 & 1.9 & 2.2 & 1.5 & 1.8 & 2.3 & 2.5 & 2.0 & 1.7 & 2.6 \\
\hline Other & 12.5 & 12.5 & 12.5 & 12.7 & 11.1 & 11.8 & 13.4 & 12.5 & 12.0 & 12.0 & 11.5 & 13.5 & 12.7 & 12.4 & 14.1 \\
\hline \multicolumn{16}{|l|}{ Region } \\
\hline Eastern & 7.1 & 7.4 & 6.9 & 7.5 & 6.5 & 7.2 & 6.6 & 7.3 & 6.7 & 7.2 & 7.0 & 7.3 & 7.0 & 7.8 & 7.3 \\
\hline $\begin{array}{l}\text { Ontario and } \\
\text { Quebec }\end{array}$ & 65.3 & 65.2 & 65.4 & 65.7 & 67.3 & 66.7 & 67.1 & 67.2 & 66.5 & 66.0 & 63.9 & 65.1 & 64.1 & 60.0 & 63.5 \\
\hline $\begin{array}{l}\text { Alberta and } \\
\text { Saskatchewan }\end{array}$ & 14.7 & 14.7 & 14.6 & 13.8 & 14.9 & 13.8 & 14.2 & 14.1 & 13.9 & 15.0 & 14.6 & 14.7 & 14.0 & 17.3 & 16.2 \\
\hline British Columbia & 12.9 & 12.7 & 13.1 & 13.0 & 11.3 & 12.3 & 12.0 & 11.4 & 13.0 & 11.8 & 14.5 & 13.0 & 14.8 & 14.8 & 13.0 \\
\hline \multicolumn{16}{|l|}{ Spending money, \$ } \\
\hline 0 & 20.0 & 19.7 & 20.4 & 25.0 & 27.7 & 24.8 & 26.9 & 21.1 & 21.5 & 20.1 & 18.1 & 14.9 & 17.1 & 11.5 & 10.9 \\
\hline $1-20$ & 30.5 & 30.8 & 30.2 & 40.8 & 41.5 & 38.2 & 37.6 & 33.6 & 34.0 & 30.5 & 29.2 & 21.9 & 21.7 & 19.4 & 16.9 \\
\hline$\geq 21$ & 30.1 & 29.0 & 31.1 & 12.8 & 11.9 & 14.1 & 16.8 & 22.3 & 26.6 & 29.7 & 34.6 & 44.7 & 42.6 & 51.4 & 55.2 \\
\hline Not stated & 19.4 & 20.6 & 18.2 & 21.3 & 18.9 & 22.9 & 18.8 & 23.0 & 17.8 & 19.6 & 18.1 & 18.6 & 18.6 & 17.7 & 17.1 \\
\hline \multicolumn{16}{|l|}{ Smoking status } \\
\hline Never smoker ${ }^{*}$ & 86.2 & 87.3 & 85.2 & 97.8 & 95.8 & 93.9 & 93.4 & 88.6 & 88.4 & 85.2 & 84.5 & 81.1 & 78.2 & 76.6 & 70.2 \\
\hline Ever smoker† & 4.8 & 3.9 & 5.8 & 0.6 & 0.9 & 1.6 & 2.1 & 7.6 & 8.0 & 3.7 & 10.0 & 5.6 & 8.7 & 8.3 & 14.2 \\
\hline $\begin{array}{l}\text { Experimental } \\
\text { smokerł }\end{array}$ & 8.9 & 8.8 & 9.2 & 1.7 & 3.3 & 4.5 & 4.5 & 3.7 & 3.6 & 11.1 & 5.6 & 13.2 & 13.2 & 15.1 & 15.5 \\
\hline $\begin{array}{l}\text { Alcohol bingeing in } \\
\text { previous } 12 \mathrm{mo}\end{array}$ & 26.4 & 26.0 & 26.8 & 2.4 & 3.2 & 7.5 & 7.3 & 21.1 & 19.9 & 32.9 & 32.6 & 42.6 & 46.6 & 50.8 & 51.7 \\
\hline $\begin{array}{l}\text { *Had not "ever tried } \\
\text { †Had tried a cigaret } \\
\text { more whole cigarett } \\
\text { fHad never tried sm }\end{array}$ & $\mathrm{cm}$ & $\begin{array}{l}\text { g, eve } \\
\text { y the }\end{array}$ & $a f$ & $\begin{array}{l}\text { fs." } \\
\text { e qu }\end{array}$ & ns & oue & ied & ette s & ing, & ust a & uffs: & " & & loke & \\
\hline
\end{tabular}




\section{OPEN}

Research

refusal to allow the child to participate, student refusal even with parental permission or absence from school or class on the day of data collection. Of the 65812 students eligible to participate, 47203 completed the questionnaires. After sorting for completeness and valid responses, 38667 questionnaires were analyzed.

\section{Sample characteristics and prevalence estimates}

Weighted characteristics of the sample are shown in Table 1. Table 2 presents self-reported prevalence data for 5 medicinal substances and 11 nonmedicinal substances from the previous year. More than 2 in 10 young people reported abuse of at least 1 of the listed medicinal and nonmedicinal sub-

Table 2: Abuse of medicinal and non-medicinal substances in the last 12 months among youth in grades 7-12, Youth Smoking Survey, Canada, 2012

\begin{tabular}{|c|c|c|c|c|c|c|c|c|c|c|c|c|c|c|c|}
\hline \multirow[b]{2}{*}{ Substance } & \multirow{2}{*}{$\begin{array}{c}\begin{array}{c}\text { All } \\
\text { grades, } \\
\%\end{array} \\
\text { All }\end{array}$} & \multicolumn{2}{|c|}{ All grades, \% } & \multicolumn{2}{|c|}{ Grade 7, \% } & \multicolumn{2}{|c|}{ Grade 8, \% } & \multicolumn{2}{|c|}{ Grade 9, \% } & \multicolumn{2}{|c|}{ Grade $10, \%$} & \multicolumn{2}{|c|}{ Grade $11, \%$} & \multicolumn{2}{|c|}{ Grade $12, \%$} \\
\hline & & Female & Male & Female & Male & Female & Male & Female & Male & Female & Male & Female & Male & Female & Male \\
\hline \multicolumn{16}{|l|}{$\begin{array}{l}\text { Medicinal } \\
\text { substances }\end{array}$} \\
\hline Any & 5.3 & 5.8 & 4.9 & 2.6 & 2.0 & 3.9 & 3.1 & 5.8 & 3.7 & 5.4 & 4.9 & 7.6 & 6.7 & 9.7 & 9.1 \\
\hline Dextromethorphan & 2.9 & 3.0 & 2.7 & 1.8 & 1.5 & 2.2 & 2.0 & 2.4 & 1.9 & 2.7 & 2.5 & 3.8 & 3.3 & 5.4 & 5.4 \\
\hline Pain medications & 2.6 & 2.8 & 2.3 & 0.8 & 0.6 & 1.7 & 1.3 & 2.4 & 1.5 & 2.4 & 2.5 & 4.3 & 3.4 & 5.7 & 4.7 \\
\hline $\begin{array}{l}\text { Sleeping } \\
\text { medications }\end{array}$ & 1.8 & 2.0 & 1.6 & 0.9 & 0.5 & 1.0 & 0.9 & 2.0 & 0.9 & 2.0 & 1.5 & 2.8 & 1.7 & 3.5 & 4.4 \\
\hline Stimulants & 1.7 & 1.6 & 1.8 & 0.8 & 0.4 & 0.8 & 0.7 & 2.2 & 1.4 & 1.1 & 1.9 & 2.0 & 2.6 & 3.1 & 3.6 \\
\hline Sedatives & 1.0 & 0.9 & 1.1 & 0.3 & 0.1 & 0.4 & 0.6 & 0.2 & 0.7 & 1.0 & 1.3 & 1.3 & 1.6 & 2.4 & 2.5 \\
\hline \multicolumn{16}{|l|}{$\begin{array}{l}\text { Nonmedicinal } \\
\text { substances }\end{array}$} \\
\hline Any & 21.3 & 20.4 & 22.1 & 2.4 & 4.6 & 7.4 & 7.6 & 15.3 & 15.3 & 23.9 & 27.1 & 34.3 & 36.0 & 40.0 & 42.9 \\
\hline Marijuana & 20.7 & 19.8 & 21.5 & 1.7 & 4.2 & 6.8 & 6.8 & 14.4 & 14.8 & 23.3 & 26.3 & 34.0 & 35.4 & 39.7 & 42.2 \\
\hline Hallucinogens & 2.4 & 1.7 & 3.1 & 0.4 & 0.2 & 0.9 & 0.6 & 1.4 & 1.8 & 1.7 & 3.3 & 3.0 & 6.1 & 2.9 & 6.7 \\
\hline MDMA & 2.3 & 2.1 & 2.6 & 0.2 & 0.1 & 0.9 & 0.7 & 1.2 & 1.5 & 2.3 & 2.2 & 3.9 & 4.0 & 4.4 & 7.1 \\
\hline $\begin{array}{l}\text { Salvia (Salvia } \\
\text { divinorum) }\end{array}$ & 1.8 & 1.1 & 2.6 & 0.3 & 0.1 & 0.3 & 0.5 & 0.4 & 1.8 & 0.9 & 3.2 & 2.6 & 4.2 & 2.1 & 5.6 \\
\hline Cocaine & 1.7 & 1.1 & 2.2 & 0.4 & 0.4 & 0.6 & 0.6 & 0.9 & 1.1 & 1.2 & 2.4 & 1.7 & 3.8 & 1.9 & 4.9 \\
\hline Amphetamines & 1.6 & 1.2 & 1.9 & 0.4 & 0.4 & 1.3 & 0.8 & 1.2 & 1.1 & 1.4 & 2.2 & 1.8 & 3.8 & 1.0 & 3.3 \\
\hline $\begin{array}{l}\text { Spice (synthetic } \\
\text { cannabis) }\end{array}$ & 1.3 & 1.0 & 1.5 & 0.6 & 0.4 & 0.8 & 1.2 & 0.8 & 1.2 & 1.2 & 1.7 & 1.5 & 2.2 & 1.1 & 2.3 \\
\hline Solvents & 0.8 & 0.6 & 1.0 & 0.5 & 0.3 & 0.6 & 0.8 & 1.1. & 0.9 & 0.5 & 1.2 & 0.5 & 1.2 & 0.3 & 1.6 \\
\hline Heroin & 0.6 & 0.3 & 0.8 & 0.2 & 0.3 & 0.4 & 0.4 & 0.2 & 0.5 & 0.3 & 0.8 & 0.4 & 1.2 & 0.3 & 1.7 \\
\hline Bath salts* & 0.5 & 0.3 & 0.8 & 0.3 & 0.1 & 0.3 & 0.4 & 0.2 & 0.6 & 0.3 & 0.8 & 0.4 & 1.5 & 0.4 & 1.5 \\
\hline BZP/TFMPP & 0.4 & 0.2 & 0.6 & 0.0 & 0.0 & 0.1 & 0.4 & 0.4 & 0.5 & 0.3 & 0.5 & 0.4 & 0.9 & 0.2 & 1.5 \\
\hline $\begin{array}{l}\text { Medicinal } \\
\text { substance abuse } \\
\text { only }\end{array}$ & 1.7 & 2.2 & 1.3 & 1.9 & 1.5 & 2.2 & 1.9 & 2.4 & 1.4 & 1.6 & 1.4 & 2.1 & 1.0 & 2.8 & 0.6 \\
\hline $\begin{array}{l}\text { Nonmedicinal } \\
\text { substance abuse } \\
\text { only }\end{array}$ & 17.7 & 16.8 & 18.6 & 1.7 & 4.0 & 5.8 & 6.4 & 11.9 & 13.0 & 20.2 & 23.6 & 28.8 & 30.3 & 33.2 & 34.4 \\
\hline $\begin{array}{l}\text { Concurrent abuse } \\
\text { of both medicinal } \\
\text { and nonmedicinal } \\
\text { substances }\end{array}$ & 3.6 & 3.6 & 3.6 & 0.7 & 0.5 & 1.7 & 1.2 & 3.4 & 2.3 & 3.7 & 3.4 & 5.5 & 5.6 & 6.8 & 8.5 \\
\hline $\begin{array}{l}\text { Any substance } \\
\text { abuse }\end{array}$ & 23.0 & 22.6 & 23.4 & 4.3 & 6.0 & 9.7 & 9.4 & 17.7 & 16.7 & 25.5 & 28.5 & 36.5 & 37.0 & 42.9 & 43.5 \\
\hline
\end{tabular}


stances. Dextromethorphan and pain relievers or tranquilizers were the substances that were most frequently reported to have been abused in the last 12 months, followed by sleeping medicines, stimulants and sedatives (Figure 1). Of the students sampled, $17.7 \%$ abused nonmedicinal substances exclusively, $1.7 \%$ abused medicinal substances exclusively and $3.6 \%$ reported abuse of both medicinal and nonmedicinal substances.

\section{Correlates of medicinal substance abuse versus substance abstinence}

Table 3 outlines the results of multinomial regression analyses examining factors associated with medicinal, nonmedicinal and concurrent substance abuse including odds ratios. Students in higher grade levels were not more likely to report abusing medicinal substances than their younger peers. Compared with boys, girls were 1.7 times as likely to abuse medicinal substances. The only differences detected by ethnicity were between those identifying within the "Black" and "Aboriginal" categories, respectively about twice as likely to report abusing medicinal substances than those identifying as "Other."

Regional discrepancies were observed between British Columbia and Ontario and Quebec. Young people in British Columbia reported significantly more abuse of medicinal substances than their peers in Ontario and Quebec. Experimental smokers and those reporting alcohol bingeing were almost twice as likely to report medicinal substance abuse as never smokers and non-binge drinkers (Table 3).

\section{Correlates of concurrent use of medicinal and nonmedicinal substances versus abstinence}

Students in higher grade levels had an increased likelihood of self-reported concurrent abuse of medicinal and nonmedicinal substances (Table 3), with girls more likely to report participating in dual abuse of substances than boys. Differences across ethnicities were noted only between Asian youth, who reported about one-third of concurrent abuse of medicinal and nonmedicinal substances, and their peers identifying as "Black" or "Other." Black youth were twice as likely to report concurrent abuse of substances as their white counterparts.

Respondents from British Columbia were more likely to report concurrent abuse of medicinal and nonmedicinal substances than those residing in Alberta and Saskatchewan and Ontario and Quebec. Children in Alberta and Saskatchewan were about half as likely as those in the Ontario and Quebec and Eastern provinces to be dual abusers. Having spending money was associated with an increased likelihood of abusing both medicinal and nonmedicinal substances. The prevalence of comorbid use increased with greater tobacco use and binge drinking.

\section{Interpretation}

Findings from this nationally representative survey of substance abuse among Canadian youth between grades 7 and 12 suggest that $5.3 \%$ of students in this age group reported abusing medicinal substances. Dextromethorphan, a sub- stance in many cough and cold syrups, was the most widely reported medicinal substance abused, followed by pain medications, sleeping medications, stimulants and sedatives. Almost one-quarter $(23.0 \%)$ of the surveyed population reported some substance abuse.

Medicinal substance abuse was consistently more prevalent among girls than among boys at each grade level. Risk behaviour and illicit drug use showed gendered pathways. ${ }^{36}$ The elevated rates of medicinal substance abuse seen in the current study may be a reflection of beliefs that medicinal substances are safer alternatives to nonmedicinal substances, are easier to access, carry less potential for interfacing with criminal organizations or police and are perhaps more socially acceptable. ${ }^{37,38}$ Regional differences were also apparent. Youth British Columbia were more likely to abuse medicinal substances, whereas youth in Alberta and Saskatchewan were the least likely to report concurrent substance abuse. These differences may be influenced by the socioeconomic context of each of these regions. Our findings suggest a greater likelihood of adolescents abusing all substances when they have disposable income, but those receiving more than $\$ 20$ of spending money are more likely than those receiving up to $\$ 20$ each week to concurrently abuse medicinal and nonmedicinal substances. Use of tobacco and alcohol was associated with greater abuse of medicinal substances and with dual substance abuse, similar to previous research. ${ }^{39}$

Direct comparisons with national estimates from other studies are not possible owing to a lack of data. According to the Canadian Alcohol and Drug Use Monitoring Survey, which surveys Canadians aged 15 and older, abuse of dextromethorphan was not reportable among youth because less than $1 \%$ of Canadians reported its abuse, in contrast to the current estimate of $2.9 \%$. The survey was not able to report prevalence of abuse of many medicinal substances owing to high sampling variability and much smaller sample sizes among youth than the current study, sustaining a continued dearth of comparable prevalence estimates. Discrepancies between Ontario Student Drug Use and Health Survey find-

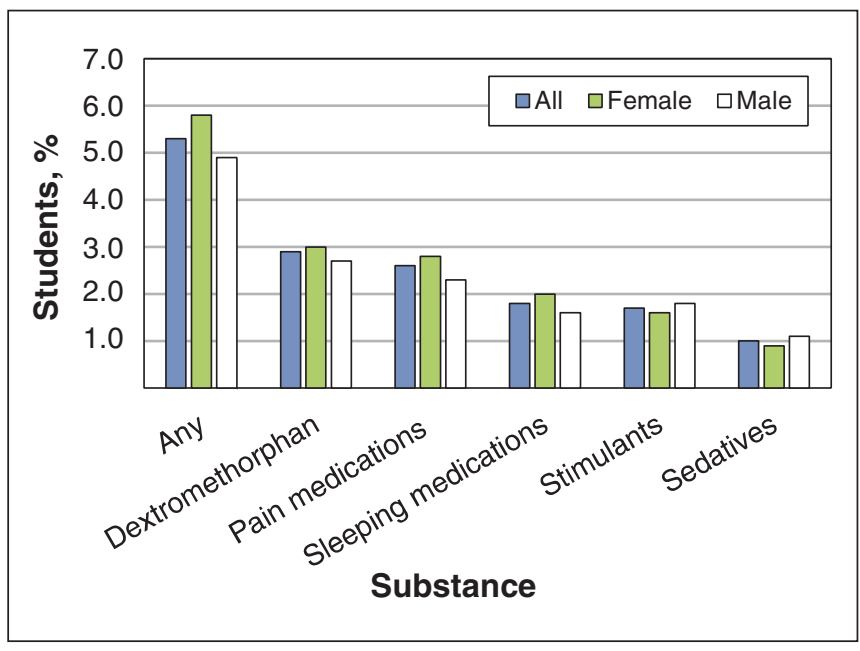

Figure 1: Abuse of medicinal substances in the previous 12 months among youth in grades 7 to $12(n=38667)$. 
Table 3: Multinomial logistic regression analyses examining factors associated with medicinal and nonmedicinal substance abuse among youth in grades 7-12, Youth Smoking Survey, Canada, 2012.

\begin{tabular}{|c|c|c|c|c|c|c|c|c|c|}
\hline \multirow{3}{*}{$\begin{array}{l}\begin{array}{l}\text { Characteristic or } \\
\text { behaviour }\end{array} \\
\begin{array}{l}\text { Grade }\left(\chi^{2}=183.060,\right. \\
p<0.001)\end{array}\end{array}$} & \multicolumn{3}{|c|}{ Medicinal substance abuse (model 1) } & \multicolumn{3}{|c|}{ Nonmedicinal substance abuse (model 2) } & \multicolumn{3}{|c|}{$\begin{array}{l}\text { Concurrent abuse of medicinal and } \\
\text { nonmedicinal substances (model 3) }\end{array}$} \\
\hline & \multicolumn{2}{|c|}{ OR $(95 \% \mathrm{Cl})$} & \multirow{2}{*}{$\frac{p^{*}}{0.4}$} & \multicolumn{2}{|c|}{ OR $(95 \% \mathrm{Cl})$} & \multirow{2}{*}{$\frac{p^{*}}{<0.001}$} & \multicolumn{2}{|c|}{ OR $(95 \% \mathrm{Cl})$} & \multirow{2}{*}{$\frac{p^{*}}{<0.001}$} \\
\hline & 1.031 & $(0.951-1.119)$ & & 1.365 & $(1.304-1.429)$ & & 1.279 & $(1.163-1.406)$ & \\
\hline \multicolumn{10}{|c|}{$\operatorname{Sex}\left(\chi^{2}=21.067, p<0.001\right)$} \\
\hline Female & 1.666 & $(1.319-2.103)$ & $<0.001$ & 0.958 & $(0.848-1.082)$ & 0.5 & 1.194 & $(1.016-1.401)$ & 0.031 \\
\hline \multicolumn{10}{|c|}{ Ethnicity $\left(\chi^{2}=168.578, p<0.001\right)$} \\
\hline \multicolumn{10}{|l|}{ Aboriginal $\mathrm{v}$. } \\
\hline Asian & 1.264 & $(0.638-2.503)$ & 1.00 & 4.351 & $(2.578-7.346)$ & $<0.001$ & 2.790 & $(0.883-8.815)$ & 0.1 \\
\hline Black & 0.827 & $(0.381-1.796)$ & 1.00 & 1.892 & $(1.062-3.372)$ & 0.02 & 0.753 & $(0.282-2.005)$ & 1.00 \\
\hline Latin & 0.882 & $(0.219-3.561)$ & 1.00 & 1.217 & $(0.683-2.169)$ & 1.00 & 1.069 & $(0.406-2.815)$ & 1.00 \\
\hline Other & 1.931 & $(1.050-3.549)$ & 0.02 & 1.808 & $(1.189-2.749)$ & $<0.001$ & 1.056 & $(0.444-2.512)$ & 1.00 \\
\hline White & 1.300 & $(0.780-2.167)$ & 1.00 & 1.991 & $(1.420-2.792)$ & $<0.001$ & 1.568 & $(0.780-3.152)$ & 0.9 \\
\hline \multicolumn{10}{|l|}{ Asian v. } \\
\hline Black & 0.654 & $(0.301-1.424)$ & 1.00 & 0.435 & $(0.284-0.667)$ & $<0.001$ & 0.270 & $(0.109-0.666)$ & $<0.001$ \\
\hline Latin & 0.698 & $(0.152-3.204)$ & 1.00 & 0.280 & $(0.172-0.452)$ & $<0.001$ & 0.383 & $(0.102-1.440)$ & 0.50 \\
\hline Other & 1.528 & $(0.808-2.888)$ & 0.8 & 0.415 & $(0.277-0.622)$ & $<0.001$ & 0.379 & $(0.147-0.973)$ & 0.04 \\
\hline White & 1.029 & $(0.535-1.978)$ & 1.00 & 0.458 & $(0.321-0.652)$ & $<0.001$ & 0.562 & $(0.226-1.396)$ & 0.9 \\
\hline \multicolumn{10}{|l|}{ Black v. } \\
\hline Latin & 1.067 & $(0.202-5.641)$ & 1.00 & 0.643 & $(0.407-1.017)$ & 0.07 & 1.420 & $(0.532-3.789)$ & 1.00 \\
\hline Other & 2.335 & $(1.111-4.908)$ & 0.01 & 0.955 & $(0.541-1.688)$ & 1.00 & 1.403 & $(0.748-2.631)$ & 1.00 \\
\hline White & 1.573 & $(0.791-3.126)$ & 0.8 & 1.052 & $(0.704-1.574)$ & 1.00 & 2.083 & $(1.040-4.171)$ & 0.03 \\
\hline \multicolumn{10}{|l|}{ Latin $\mathrm{v}}$. \\
\hline Other & 2.188 & $(0.592-8.087)$ & 1.00 & 1.485 & $(0.810-2.722)$ & 0.8 & 0.988 & $(0.398-2.451)$ & 1.00 \\
\hline White & 1.474 & $(0.412-5.265)$ & 1.00 & 1.636 & $(1.043-2.565)$ & 0.02 & 1.467 & $(0.636-3.383)$ & 1.00 \\
\hline \multicolumn{10}{|l|}{ Other v. } \\
\hline White & 0.674 & $(0.442-1.026)$ & 0.09 & 1.101 & $(0.806-1.505)$ & 1.00 & 1.485 & $(0.912-2.418)$ & 0.3 \\
\hline Region $\left(\chi^{2}=81.292, p\right.$ & 001) & & & & & & & & \\
\hline British Columbia v. & & & & & & & & & \\
\hline Ontario and Quebec & 1.427 & $(1.087-1.994)$ & 0.005 & 1.310 & $(0.983-1.746)$ & 0.08 & 1.469 & $(1.058-2.039)$ & 0.01 \\
\hline $\begin{array}{l}\text { Alberta and } \\
\text { Saskatchewan }\end{array}$ & 1.209 & $(0.909-1.609)$ & 0.5 & 1.795 & $(1.179-2.733)$ & 0.002 & 2.369 & $(1.638-3.426)$ & $<0.001$ \\
\hline Eastern provinces & 1.474 & $(1.016-2.139)$ & 0.3 & 1.243 & $(0.879-1.757)$ & 0.6 & 1.087 & $(0.747-1.579)$ & 1.00 \\
\hline Ontario and Quebec v. & & & & & & & & & \\
\hline $\begin{array}{l}\text { Alberta and } \\
\text { Saskatchewan }\end{array}$ & 0.822 & $(0.622-1.086)$ & 0.4 & 1.370 & $(0.955-1.965)$ & 0.1 & 1.612 & $(1.034-2.514)$ & 0.03 \\
\hline Eastern provinces & 1.002 & $(0.687-1.461)$ & 1.00 & 0.949 & $(0.727-1.237)$ & 1.00 & 0.740 & $(0.485-1.128)$ & 0.4 \\
\hline Alberta and Saskatchev & & & & & & & & & \\
\hline Eastern provinces & 1.219 & $(0.900-1.651)$ & 0.50 & 0.692 & $(0.508-0.943)$ & 0.01 & 0.459 & $(0.318-0.661)$ & $<0.001$ \\
\hline Spending money $\left(\chi^{2}=\right.$ & $051, p<$ & $.001), \$$ & & & & & & & \\
\hline $1-20 \mathrm{v}$ & & & & & & & & & \\
\hline$\geq 21$ & 1.120 & $(0.790-1.588)$ & 1.00 & 0.919 & $(0.748-1.130)$ & 1.00 & 0.649 & $(0.466-0.903)$ & 0.003 \\
\hline Not stated & 1.466 & $(0.930-2.313)$ & 0.2 & 1.615 & $(1.265-2.062)$ & $<0.001$ & 1.419 & (0.989-2.035) & 0.06 \\
\hline Zero & 1.470 & $(0.838-2.581)$ & 0.4 & 1.510 & (1.183-1.929) & $<0.001$ & 1.100 & $(0.704-1.719)$ & 1.00 \\
\hline$\geq 21 \mathrm{v}$ & & & & & & & & & \\
\hline Not stated & 1.309 & $(0.817-2.098)$ & 0.8 & 1.756 & $(1.371-2.250)$ & $<0.001$ & 2.187 & $(1.412-3.386)$ & $<0.001$ \\
\hline Zero & 1.312 & $(0.718-2.400)$ & 1.00 & 1.643 & $(1.254-2.152)$ & $<0.001$ & 1.695 & $(1.059-2.712)$ & 0.02 \\
\hline Not stated $\mathrm{v}$. & & & & & & & & & \\
\hline Zero & 1.003 & $(0.552-1.823)$ & 1.00 & 0.935 & $(0.734-1.191)$ & 1.00 & 0.775 & $(0.490-1.227)$ & 0.9 \\
\hline Smoking $\left(\chi^{2}=1241.32\right.$ & $<0.001)$ & & & & & & & & \\
\hline Experimental v. & & & & & & & & & \\
\hline Ever smoker & 2.759 & $(0.672-11.324)$ & 0.3 & 0.603 & $(0.396-0.918)$ & 0.01 & 0.299 & $(0.190-0.470)$ & $<0.001$ \\
\hline Never smoker & 1.851 & $(1.037-3.303)$ & 0.03 & 8.535 & $(7.031-10.362)$ & $<0.001$ & 17.161 & (11.879-24.791) & $<0.001$ \\
\hline Ever smoker v. & & & & & & & & & \\
\hline Never smoker & 0.671 & $(0.181-2.483)$ & 1.00 & 14.151 & (9.571-20.924) & $<0.001$ & 57.462 & (36.009-91.695) & $<0.001$ \\
\hline $\begin{array}{l}\text { Ever binged on alcohol } \\
\left(\chi^{2}=542.554\right. \\
p<0.001)\end{array}$ & 1.732 & $(1.336-2.245)$ & $<0.001$ & 7.266 & $(6.120-8.626)$ & $<0.001$ & 9.998 & $(7.202-13.880)$ & $<0.001$ \\
\hline Reference category & & Substance abs & & & : Substance abst & & & Substance absti & \\
\hline
\end{tabular}


ings for other substances and the ones reported here reflect differences in survey designs. Where the Youth Smoking Survey explicitly asks about consuming substances to "get high and not for medical reasons," the Ontario Student Drug Use and Health Survey asks children whether they have used particular substances without a prescription or without a doctor telling them to take it; the resulting findings from the Ontario Student Drug Use and Health Survey are not necessarily indicative of the way the United States Food and Drug Administration has operationalized abuse but simply a way they have chosen to categorize a type of substance misuse. ${ }^{40}$

\section{Limitations}

Although this study is particularly strong because of its generalizability among Canadian youth, it has several limitations common to survey research, including nonresponse, issues with self-reporting and potential sample bias. The 2012-2013 Youth Smoking Survey captures a nationally representative sample of boys and girls attending mainstream school settings; however, it excludes youth that were not or could not be present on the day the survey was administered, does not account for on-reserve schools, alternative schools where high-risk youth may attend and excluded regions where there may be greater proportions of at-risk youth. Thus, the current estimates of medicinal substance abuse may conservatively estimate the current prevalence of substance abuse among youth today. In addition, the survey defines binge drinking as 5 or more drinks, whereas the US Centers for Disease Control and Prevention defines it as 4 or more drinks for women or 5 or more for men. ${ }^{41}$

\section{Conclusion}

The current findings suggest that almost one-quarter of Canadian youth from grades 7 to 12 reported abusing medicinal or nonmedicinal substances. Cough and cold syrups and pain medications were the most widely abused of the medicinal substances. These analyses contribute important information to the fragmented literature that exists today about substance abuse in general.

\section{References}

1. Foster K, Spencer D. 'It's just a social thing': drug use, friendship and borderwork among marginalized young people. Int J Drug Policy 2013;24:223-30.

2. Kirst M, Mecredy G, Chaiton M. The prevalence of tobacco use co-morbidities in Canada. Can J Public Health 2013;104:e210-5.

3. Whitesell NR, Asdigian NL, Kaufman CE, et al. Trajectories of substance use among young American Indian adolescents: patterns and predictors. $J$ Youth Adolesc 2014;43:437-53.

4. Werb D, Kerr T, Buxton J, et al. Crystal methamphetamine and initiation of injection drug use among street-involved youth in a Canadian setting. CMAJ 2013;185:1569-75

5. Gruskin S, Plafker K, Smith-Estelle A. Understanding and responding to youth substance use: the contribution of a health and human rights framework. Am J Public Health 2001;91:1954-63.

6. Canadian drug summary: prescription opioids. Ottawa: Canadian Centre on Substance Abuse; 2013; Available: http://nnadaprenewal.ca/wp-content/ uploads/2013/03/3-CCSA-Prescription-Opioids-2013-en.pdf (accessed 2013 Nov. 10)

7. Controlled Drugs and Substances Act, SC 1996, c 19. Available: http://canlii. ca/t/52hkj (accessed 2015 Oct. 21)

8. Combating misuse and abuse of prescription drugs: Q \& A with Michael Klein, PhD. Silver Spring (MD): United States Food and Drug Administration; 2010. Available: www.fda.gov/ForConsumers/ConsumerUpdates/ucm220112.htm (accessed 2014 Aug. 28)

9. Rehm J, Gnam W, Popova S, et al. The costs of alcohol, illegal drugs, and tobacco in Canada, 2002. J Stud Alcohol Drugs 2007;68:886-95

10. Pirie T, Jesseman R, Di Gioacchino L; National Treatment Indicators Working Group. National Treatment Indicators Report: 2010-2011 Data. Ottawa: Canadian Centre on Substance Abuse; 2013; Available: www.ccsa.ca/Resource\%20 Library/NTS-2014-National-Treatment-Indicators-Report-en.pdf (accessed 2014 Sept. 24).

11. Fischer B, Argento E. Prescription opioid related misuse, harms, diversion an interventions in Canada: a review. Pain Physician 2012;15(Suppl):ES191-203.

12. Fischer B, Jones W, Rehm J. Trends and changes in prescription opioid analgesic dispensing in Canada 2005-2012: an update with a focus on recent interventions. BMC Health Serv Res 2014;14:90.

13. Fischer B, Rehm J, Goldman B, et al. Non-medical use of prescription opioids and public health in Canada: An urgent call for research and interventions development. Can J Public Health 2008;99:182-4.

14. Fischer B, Ialomiteanu A, Boak A, et al. Prevalence and key covariates of nonmedical prescription opioid use among the general secondary student and adult populations in Ontario, Canada. Drug Alcohol Rev 2013;32:276-87.

15. Fischer B, Gooch J, Goldman B, et al. Non-medical prescription opioid use, prescription opioid-related harms and public health in Canada: an update 5 years later. Can J Public Health 2014;105:e146-9.

16. Longo LP, Parran T Jr, Johnson B, et al. Addiction: part 11. Identification and management of the drug-seeking patient. Am Fam Physician 2000;61:2401-8.

17. Antoniou T, Juurlink DN. Dextromethorphan abuse. CMAJ 2014;186:E631.

18. Amaladoss A, Brien SO. Cough syrup psychosis. CJEM 2011;13:53-6.

19. Gershman JA, Fass AD. Dextromethorphan abuse: a literature review. J Pharm Technol 2013;29:66-71.

20. Miller SC. Dextromethorphan psychosis, dependence and physical withdrawal Addict Biol 2005;10:325-7.

21. Boak A, Hamilton HA, Mann RE. Drug use among Ontario students, 19772013: Detailed OSDUHS findings. (CAMH Research Document Series No. 36). Toronto: Centre for Addition and Mental Health; 2013. Available: www.camh.ca/ en/research/news_and_publications/ontario-student-drug-use-and-health-survey/ Documents/2013\%20OSDUHS\%20Docs/2013OSDUHS_Detailed_ DrugUseReport.pdf (accessed 2015 Oct. 2)

22. Blakemore SJ, Choudhury S. Development of the adolescent brain: implications for executive function and social cognition. J Child Psychol Psychiatry 2006; 47:296-312.

23. Hare TA, Tottenham N, Galvan A, et al. Biological substrates of emotional reactivity and regulation in adolescence during an emotional go-nogo task. Biol Psychiatry 2008;63:927-34.

24. Casey BJ, Jones RM, Hare TA. The adolescent brain. Ann N Y Acad Sci 2008; 1124:111-26.

25. Steinberg L. Adolescence. 6th ed. New York: McGraw-Hill; 2002.

26. Burghardt LC, Ayers JW, Brownstein JS, et al. Adult prescription drug use and pediatric medication exposures and poisonings. Pediatrics 2013;132:18-27.

27. Dalsgaard S, Mortensen PB, Frydenberg M, et al. ADHD, stimulant treatment in childhood and subsequent substance abuse in adulthood - a naturalistic longterm follow-up study. Addict Behav 2014;39:325-8.

28. Pasch KE, Velazquez CE, Cance JD, et al. Youth substance use and body composition: Does risk in one area predict risk in the other? J Youth Adolesc 2012;41:14-26.

29. Huang DYC, Lanza HI, Wright-Volel K, et al. Developmental trajectories of childhood obesity and risk behaviors in adolescence. J Adolesc 2013;36:139-48.

30. Trenz RC, Scherer M, Harrell P, et al. Early onset of drug and polysubstance use as predictors of injection drug use among adult drug users. Addict Behav 2012;37:367-72

31. de Graaf R, Radovanovic M, Van Laar M, et al. Early cannabis use and estimated risk of later onset of depression spells: epidemiologic evidence from the population-based World Health Organization World Mental Health Survey Initiative. Am J Epidemiol 2010;172:149-59.

32. Schmid B, Hohm E, Blomeyer D, et al. Concurrent alcohol and tobacco use during early adolescence characterizes a group at risk. Alcohol Alcohol 2007;42:219-25.

33. Burkhalter R, Cumming T, Rynard V, Manske S. 2012/2013 Youth Smoking Survey microdata user guide. Waterloo $(\mathrm{ON})$ : Propel Centre for Population Health Impact, University of Waterloo, 2013:1-47. Available: https://uwaterloo.ca/ canadian-student-tobacco-alcohol-drugs-survey/sites/ca.canadian-student-tobacco -alcohol-drugs-survey/files/uploads/files/yss12_user_guide_en_v5_20131231.pdf (accessed 2015 Apr. 3).

34. Summary of results of the Youth Smoking Survey. Ottawa: Health Canada; 2014. Available: www.hc-sc.gc.ca/hc-ps/tobac-tabac/research-recherche/ stat/_survey-sondage_2012-2013/result-eng.php (accessed 2014 Aug. 3).

35. Hammond D, Ahmed R, Sae Yang W, et al. Illicit substance use among Canadian youth: trends between 2002 and 2008. Can 7 Public Health 2011; 102:7-12.

36. Poole N, Dell CA. Girls, women and substance use. Ottawa: Canadian Centre on Substance Abuse. 2005:1-16.

37. Ford JA. Nonmedical prescription drug use among adolescents: the influence of bonds to family and school. Youth Soc 2009;40:336-52

38. Currie CL, Wild TC. Adolescent use of prescription drugs to get high in Canada. Can 7 Psychiatry 2012;57:745-51.

39. Acquavita SP, McClure EA, Hargraves D, et al. Environmental tobacco smoke exposure among smokers and non-smokers receiving outpatient substance abuse treatment. Addict Behav 2014;39:1718-22. 
40. Student Drug Use Surveys Working Group. The value of student alcohol and drug use surveys: the influence of student survey data on policies, programs and practice across Canada. Ottawa: Canadian Centre on Substance Abuse; 2013. Available: www.ccsa.ca/Resource\%20Library/SDUS-Value-en.pdf (accessed 2014 Sept. 25).

41. National Institute of Alcohol Abuse and Alcoholism (NIAAA). NIAAA Council approved definition of binge drinking. NIAAA Newsletter 2004;3:3.

Affiliation: School of Public Health \& Health Systems, University of Waterloo, Waterloo, Ont.

Contributors: All of the listed authors fulfill International Committee of Medical Journal Editors' authorship criteria.

Funding: The Youth Smoking Survey is a product of the pan-Canadian capacity-building project funded through a contribution agreement between Health Canada and the Propel Centre for Population Health Impact from 2004 to 2007 and a contract between Health Canada and the Propel Centre for Population Health Impact from 2008 to 2011. The Youth Smoking Survey consortium includes Canadian tobacco control researchers from all provinces and provided training opportunities for university students at all levels. The views expressed herein do not necessarily represent the views of Health Canada. This study was supported by a Canadian Institutes of Health Research New Investigator Award (D. Hammond), a Canadian Cancer Society Research Institute Junior Investigator Award (D. Hammond), a Chair in Applied Public Health funded by the Public Health Agency of Canada in partnership with the Canadian Institutes of Health Research (D. Hammond), and also partially funded by Canadian Cancer Society Grant no. 701019 (S. Manske).

Supplemental information: For reviewer comments and the original submission of this manuscript, please see www.cmajopen.ca/content/3/4/E387/suppl/DC1 